\title{
Primary Decompressive Craniectomy: Effects on Neurocritical Care Management, Long-Term Neuro- logic Status and Mortality
}

Bilotta $\mathrm{F}^{1^{*}}$, Lolli $\mathrm{S}^{1}$, Schooley $\mathrm{JN}^{2}$, Soghomonyan $\mathrm{S}^{2}$, Bergese $\mathrm{SD}^{2,3}$, Santoro A ${ }^{1}$, Roberto Delfini ${ }^{1}$, Giovanni Rosa ${ }^{1}$

${ }^{1}$ Department of Anesthesiology, Critical Care and Pain Medicine, Neurosurgery, "Sapienza” University of Rome, Rome, Italy.

${ }^{2}$ Department of Anesthesiology, Neuroanesthesia, The Ohio State University, Columbus, OH, USA.

${ }^{3}$ Department of Neurological Surgery, The Ohio State University, Columbus, OH, USA.

\begin{abstract}
Objectives: Decompressive craniectomy (DC) is a surgical therapy used to treat patients foreseen to be at risk for high intracranial pressure (ICP). In this retrospective case control study, mortality, ICP values, neurocritical care (NCC) management necessity, and long-term neurologic status were examined in patients treated with DC and in a matched control group.

Methods: The primary end-points were all-cause mortality and functional status of NCC patients both at discharge and at a 6 month follow-up. Secondary end-points were ICP values at established time points and the use of advanced NCC therapies.

Patients who underwent primary DC were matched with individuals with similar demographic and pre-intervention ICP values who had been treated with standard NCC management alone.

Results Neurologic status outcome at discharge and the 6 month follow-up was significantly better in patients treated with DC compared to those in the control group: Glasgow Outcome Scale (GOS) 4-5 in 15 versus 5 patients; $p=0.033$, and 16 versus 6 patients, $\mathrm{p}=0.033$. Mortality at 6 months was similar in the study groups $(10$ versus 16 patients; $\mathrm{p}=0.212)$. ICP values were similar at NCC admission but were better controlled 24 hours after DC than in the control group. Fewer patients treated with DC needed advanced NCC medical therapies.

Conclusions: DC is an effective way to normalize ICP levels while reducing the need for aggressive medical therapies. Survival rate and neurological outcome in patients treated with DC were found to be better than in those receiving only medical treatment.
\end{abstract}

Keywords: Primary Decompressive Craniectomy; Neurocritical Care Patients; Elevated ICP Management; Neurologic Status; Neurosurgical Mortality.

\section{*Corresponding Author:}

Federico Bilotta, M.D., Ph.D.,

Professor of Clinical Anesthesiology at the Albert Einstein College of Medicine, The Bronx, New York, NY, USA. And Department of Anesthesiology, Critical Care and Pain Medicine, "Sapienza" University Rome, Viale Acherusio 16, Rome 00199, Italy.

Tel/Fax: +39068608273

E-Mail: bilotta@tiscali.it

Received: January 06, 2015

Accepted: February 27, 2015

Published: March 02, 2015

Citation: Bilotta F et al., (2015) Primary Decompressive Craniectomy: Effects on Neurocritical Care Management, Long-Term Neurologic Status and Mortality. Int J Anesth Res. 3(2), 79-84. doi: http://dx.doi. org $/ 10.19070 / 2332-2780-1500020$

Copyright: Bilotta $\mathbf{F}^{\circ}$ 2015. This is an open-access article distributed under the terms of the Creative Commons Attribution License, which permits unrestricted use, distribution and reproduction in any medium, provided the original author and source are credited.

\section{Introduction}

Intracranial hypertension, defined as an increase in ICP above $20 \mathrm{mmHg}$ for longer than five minutes in non-stimulated patients, is due to an increase in volume of one of the determinants of ICP: brain tissue, blood volume, and cerebrospinal fluid (CSF) [1-

3]. The most dangerous consequence of this scenario is the acute onset of cerebral herniation associated with rapid worsening of neurological status and signs of brain stem compression [4] Clinical priority in the treatment of these patients is to relieve high ICP while maintaining adequate cerebral perfusion pressure (CPP) and cerebral blood flow (CBF) [5]. Standard care for patients at risk for high ICP involves lifting the patient's head 30 degrees (Fowler's position), oxygen therapy to obtain a $\mathrm{PaO}_{2}$ greater than $100 \mathrm{mmHg}$, fluid resuscitation with $0.9 \%$ isotonic saline solution, control of water and electrolyte balance, as well as aggressive treatment of hyperthermia and hyperglycemia. Pharmacologic therapies include the administration of central analgesics and sedative-hypnotics, induction of barbiturate coma to diminish the brain metabolism with resultant decrease in ICP, prophylactic 
anti-seizure therapy [6-8], and diuretics including mannitol and/ or hypertonic saline solution to reduce brain edema [1, 9]. Neurocritical care management of intracranial hypertension encompasses the use of aforementioned and other therapeutic measures including hemodynamic management of CPP with vasoactive drugs, controlled hyperventilation to maintain $\mathrm{PaCO}_{2}$ values $<35$ $\mathrm{mmHg}$, ventricular drainage, and therapeutic hypothermia $[1,10]$. However, these therapies can have significant drawbacks with regards to brain perfusion and neuronal metabolism.

DC is a surgical therapy used to treat patients with high ICP refractory to standard therapy (secondary DC) or those foreseen to be at risk for high intractable ICP (primary DC) [11]. The rationale for this approach consists of the conversion of a closed compartment into an open system with increased capacity in order to limit the negative effects of sudden increases in the volume of brain components $[12,13]$. Despite the potential of this approach, its clinical efficacy is controversial $[11,13,14]$.

The aim of this retrospective case control study was to report the effects of primary DC on the mortality rate and long term functional status (Glasgow Outcome Scale at discharge and 6th months) of patients with increased ICP. We also investigated the need for NCC therapies (vasoactive drugs, controlled hyperventilation, barbiturate coma) in patients who underwent DC compared to those medically managed alone for elevated ICP.

\section{Methods}

The protocol and consent forms were approved by the Institutional Review Board for Human Research of the University of Rome "La Sapienza". From January 2009 to January 2012, demographic and clinical data were collected and analyzed retrospectively comparing patients treated with primary DC and matched controls treated with standard medical therapies alone. Patients with severe traumatic brain injury, massive stroke or hemorrhage, bilateral fixed mydriasis, and a Glasgow Coma Scale (GCS) of 3 or those with significant comorbidities were excluded from the study because of the overall poor prognosis independent of therapeutic interventions.

Upon admission to the neurosurgical intensive care unit, ICP monitoring devices were installed in all cases. Intra-parenchymal catheters (Codman ICP Monitoring System, ICP Express) were placed on the most damaged side of the brain or on the right hemisphere in cases of diffuse bilateral damage. A neurological examination with GCS was conducted in each patient when metabolic and hemodynamic stability was restored and before any pharmacological sedation was administered. ICP values were assessed on admission to the neurosurgical ICU, before decompression, at $24 \mathrm{~h}$ and $72 \mathrm{~h}$ after decompression in the DC group and at corresponding time points in the control patients. All patients were continuously monitored (Siemens SC 7000, Sweden) with a 3-lead ECG to measure heart rate (HR), invasive mean arterial pressure (MAP), arterial oxygen saturation $\left(\mathrm{SpO}_{2}\right)$ and end-tidal $\mathrm{CO}_{2}\left(\mathrm{ETCO}_{2}\right)$. Patients were intubated and mechanical ventilation (synchronized intermittent mandatory ventilation (SIMV) modality with tidal volume of $7 \mathrm{ml} / \mathrm{kg}$ ) was adjusted for respiratory rate, an inspiratory fraction of $\mathrm{O}_{2}$ ensuring a $\mathrm{PaO}_{2}$ between 100 and $200 \mathrm{mmHg}$, and an end-tidal volume of $\mathrm{CO}_{2}\left(\mathrm{EtCO}_{2}\right)$ between 35 and $40 \mathrm{mmHg}$. Positive end-expiratory pressure (PEEP, $7 \pm 3 \mathrm{~cm}$ $\mathrm{H}_{2} 0$ ) was utilized as tolerated in patients to optimize ventilatory pattern and reduce the incidence of pulmonary infections. Each patient received fluid resuscitation therapy with isotonic saline solution $(1 \mathrm{ml} / \mathrm{kg} / \mathrm{h})$ and, whenever indicated, blood replacement with appropriate blood components. In patients with fever ( $\mathrm{T}>38$ $\left.{ }^{\circ} \mathrm{C}\right)$, paracetamol (1g every 6-8 hours) or diclofenac $(0.05 \mathrm{mg} /$ $\mathrm{kg} / \mathrm{h}$ ) was continuously infused while in cases of refractory fever, an electric cooling device (fan or cooling blanket) was used. To reduce the cerebral metabolic rate and better control the ICP, propofol $(3 \mathrm{mg} / \mathrm{kg} / \mathrm{h})$ and remifentanil $(0.25 \mathrm{mcg} / \mathrm{kg} / \mathrm{min})$ were continuously infused. In cases where this was insufficient, midazolam $(0.1-0.2 \mathrm{mg} / \mathrm{kg} / \mathrm{h})$ was added.

In patients undergoing primary DC, craniectomy method was selected by neurosurgeons based on CT scan indications. Unilateral hemicraniectomy (fronto-temporo-parietal flap) was performed when hemispheric edema was evident while bilateral craniectomy (bifrontal flap) was performed in cases of diffuse edema. In both craniectomy methods, a large bone flap was removed followed by duraplasty to allow further expansion of the brain. The exposed brain was then covered by either a homologous pericranial autograft or by a Gortex graft. The bone flap was stored in a freezer at $-18^{\circ} \mathrm{C}$ until reinsertion, which occurred in all surviving patients an average of four months after the DC (range 1-7 months).

Patients who underwent primary DC were matched with individuals who possessed similar demographic and pre-intervention ICP values who had been treated with standard NCC management alone to create a control group. NCC management of elevated ICP (used in combination with DC in the study group or alone in the control group) utilized a sequential approach: mannitol $18 \%$ via intermittent intravenous bolus at a dose of $0.25-1 \mathrm{~g} / \mathrm{kg}$ (and, if necessary for additional diuresis, furosemide $20-40 \mathrm{mg} /$ day), controlled hyperventilation to achieve an $\mathrm{EtCO}_{2}$ between 28 and $30 \mathrm{mmHg}$, and barbiturate coma with thiopental (3-5 mg/kg/h) to achieve a burst suppression state documented with EEG monitoring. A MAP> $90 \mathrm{mmHg}$ was maintained to ensure a cerebral perfusion pressure (CPP) of no less than $70 \mathrm{mmHg}$. According to standard practice, if CPP fell below $70 \mathrm{mmHg}$, a continuous infusion of norepinephrine $(0.03-0.1 \mathrm{mcg} / \mathrm{kg} / \mathrm{min})$ was started and titrated to achieve this target.

The primary endpoints were all-cause mortality and patient functional status both at discharge and at a 6-month follow-up. Neurological functional status was evaluated using the Glasgow Outcome Scale [15]. A GOS score of 4 (moderately disabled) or 5 (independent) was considered favorable while a GOS of 3 (severe disability) or 2 (persistent vegetative state) was considered unfavorable. Functional evaluation follow-up at 6 -months was recorded by a single expert neurologist after interviewing the patients as well as their relatives, attending physician, family physician doctor, or rehabilitation physician. These interviews were conducted in the outpatient setting by the physician in charge. Secondary endpoints were ICP values at the established time points and overall utilization and escalation of ICP-modulating NCC therapies in the DC and control groups including therapeutic hyperventilation and PEEP optimization as well as the use of barbiturate coma, furosemide, and norepinephrine.

\section{Statistical Analysis}

All values are reported as a mean $\pm \mathrm{SD}$ or as a percentage. A paired-samples $t$-test was used to compare ICP values before and after intervention. Likewise, this test also was used to compare hemodynamic variables and utilization of NCC interventions 
between the study groups. Percentage differences were evaluated with a chi-square or Fisher's exact test. SPSS 12.0 for Windows was used for the analysis. A p value $<0.05$ was considered statistically significant.

\section{Results}

From January 2008 to January 2012, 66 patients were treated with primary DC for ICP elevation but only 34 met study inclusion requirements. Thirty-four additional patients who possessed similar demographic parameters (weight, height, BMI, gender, race), comorbid conditions, as well as pre-intervention elevated ICP values, who were treated with standard medical therapies alone, were matched as a control group. The patients' age, gender, injury type and GCS values at admission are shown in Table 1.

There was no significant difference in mortality rate between the two groups (29.4\% (10 patients) in the DC group vs. $47 \%$ (16 patients $)$ in the standard care group $(\mathrm{p}=0.212))$. Of note, the majority of deaths occurred in the first $48 \mathrm{~h}$ after admission to the ICU. The functional outcome of all surviving patients also was assessed. In the DC group, 62.5\% (15 patients) had a good outcome at discharge (GOS 4-5) and 37.5\% (9 patients) had a poorer one (GOS 2-3). In the standard care group, $27.7 \%$ (5 patients) had a good outcome and $72.2 \%$ (13 patients) had a poorer one. These results were statistically significant demonstrating that DC patients had better outcomes at the time of discharge $(p=0.033)$. At the 6 month follow-up, data were similar, and the significant difference between the two groups was maintained. In the DC group, 66.6\% (16 patients) had a good outcome versus 33.3\% (8 patients) of poorer outcome, while only $33.3 \%$ (6 patients) in the standard care group had a good outcome $(\mathrm{p}=0.033)$.

The ICP values on admission were similar in both groups $(28.7 \pm$ 2.4 vs. $27.2 \pm 3.5, \mathrm{p}>0.05)$, however, $24 \mathrm{~h}$ after decompression, the ICP values were significantly lower in the DC group (11.2 \pm 2.4 vs. $17.5 \pm 3.2, \mathrm{p}<0.0005)$. At $72 \mathrm{~h}$ after decompression, the difference was smaller but remained statistically significant (13.5 \pm 3.6 vs. $16.7 \pm 2.3 ; \mathrm{p}<0.0005)$. All patients received anti-edema therapy by infusion of mannitol four times daily, but only $11 \mathrm{pa}-$ tients $(32.3 \%)$ in the DC group required addition of furosemide, significantly less than the 21 patients $(61.7 \%)$ who required furosemide in the standard care group ( $p=0.028)$. Additionally, the duration of furosemide therapy was shorter in the DC group, avoiding collateral effects of the drug (96 $\pm 48 \mathrm{~h}$ vs. $168 \pm 72 \mathrm{~h}$; $\mathrm{p}=0.006)$.

Some patients required an infusion of norepinephrine to ensure an adequate CPP. There was a significant difference between the study groups both in administered doses and infusion time. In the DC group, fewer patients needed norepinephrine infusion compared to patients not treated with DC: 14 DC patients $(41.7 \%)$ required an infusion of norepinephrine of $0.05 \pm 0.02 \mathrm{mcg} / \mathrm{kg} /$ min while 23 patients $(67.6 \%)$ in the standard care group required an infusion of $0.1 \pm 0.05 \mathrm{mcg} / \mathrm{kg} / \mathrm{min}(\mathrm{p}=0.001)$. Additionally, in patients in the DC group, lower rates of norepinephine infusion were required to maintain adequate CPP. The infusion time in the DC group also was lower than that of the standard care group (21 \pm 8 h vs. $36 \pm 6$ h; $\mathrm{p}<0.0001)$.

There were significant differences between the study groups in the use of therapeutic hyperventilation and barbiturate coma. Therapeutic hyperventilation was used less frequently in the DC group, 8 patients $(23.5 \%)$ versus 18 patients $(52.9 \%)(p=0.024)$. The use of barbiturate coma followed the same trend: 4 patients $(11.7 \%)$ in DC group versus 12 patients $(35.2 \%)$ in the standard care group $(p=0.043)$. Additionally, the duration of barbiturate coma was significantly lower in the DC group, $22 \pm 6$ h vs. $35 \pm$ $6 \mathrm{~h}(\mathrm{p}=0.03)$.

The use of PEEP $\left(7 \pm 3 \mathrm{~cm} \mathrm{H}_{2} \mathrm{O}\right)$ to optimize patient ventilatory pattern and reduce the incidence of pulmonary infections was especially useful in the DC group as more patients were able to tolerate it compared to the standard care group, 26 patients $(76.4 \%)$ vs. 16 patients $(47 \%)$ respectively $(p=0.024)$. This difference was

Table 1. Demographic and clinical data of patients treated with decompressive craniectomy and with standard care therapy

\begin{tabular}{|c|l|l|}
\hline \multicolumn{1}{|c|}{ Data } & \multicolumn{1}{|c|}{ DC } & \multicolumn{1}{c|}{ CG } \\
\hline Number of patients & 34 & 34 \\
\hline Aget & $47 \pm 9$ & $52 \pm 8$ \\
\hline Male/Female & $20 / 14$ & $24 / 10$ \\
\hline \multirow{5}{*}{ Type of Injury } & TBI 15 & TBI 12 \\
\cline { 2 - 3 } & SDE 4 & SDE 5 \\
\cline { 2 - 3 } & SAH 7 & SAH 5 \\
\cline { 2 - 3 } & ICH 5 & ICH 9 \\
\cline { 2 - 3 } & AIS 3 & AIS 4 \\
\hline \multirow{5}{*}{ GCS at admission } & GCS 3-8: 9 & GCS 3-8: 8 \\
\cline { 2 - 3 } & GCS 9-12: 15 & GCS 9-12: 14 \\
\cline { 2 - 3 } & GCS 13-15: 10 & GCS 13-15: 12 \\
\hline
\end{tabular}

$\dagger$ The values are expressed in mean $\pm \mathrm{SD}$

DC=Decompressive Craniectomy; CG=Control Group; TBI: Traumatic Brain Injury; SDH: Subdural Hematoma; SAH: Subarachnoid Hemorrhage; ICH: Intracerebral Hemorrhage; AIS: Acute Ischemic Stroke; GCS: Glasgow Coma Scale. 
likely due to the effect of PEEP on intracranial pressure, limiting its use in patients not undergoing a craniectomy.

\section{Discussion}

This study demonstrated that patients treated with DC had a more favorable neurological outcome than patients treated by medical therapy alone. This was evident both at discharge and after six months. Moreover, post-therapy ICP was significantly lower in patients treated with DC, and patients in the DC group needed fewer intensive medical therapies such as hyperventilation, barbiturate coma, diuretic therapy and catecholamine administration thus reducing the incidence of associated adverse effects and neurotoxicity. This study supports published evidence describing better ICP control, less intensive therapy, and improvement of neurological outcome in patients who undergo DC [11, 13].

\section{Hemodynamic Management}

The principal objective of DC is to normalize ICP and restore a favorable level of CBF [11-13]. Reduction of elevated ICP following DC can be explained by the increase in intracranial compliance with establishment of a more favorable pressure/volume ratio which promotes restoration of CBF. Cerebral blood flow increases bilaterally even if the decompression is unilateral; moreover, there are no substantial differences in blood flow following a bilateral decompression. Doppler flowmetry studies show an immediate and stable normalization of global cerebral hemodynamics for, at least, the first 48 hours following the completion of the procedure [16]. This suggests that decompression allows for rapid recruitment of capillaries and microcirculation zones that were compressed and poorly functioning in intracranial hypertensive conditions. In patients in whom ICP does not normalize after DC, the mortality rate is extremely high indicating a major and progressive extension of ischemic cerebral damage [17].

In patients with elevated ICP, clinical evidence suggests that CPP should be maintained at a minimum of $70 \mathrm{~mm} \mathrm{Hg}$. It is therefore imperative to prevent systemic arterial hypotension by assuring a MAP of no less than $90 \mathrm{~mm} \mathrm{Hg}[1,18]$. The maintenance of elevated hemodynamic values often demands continuous infusion of vasoactive amines and/or volume overload [19]. This approach is potentially dangerous as it can increase cerebral edema or cause cardiac decompensation, pulmonary edema, metabolic acidosis and coagulopathy [20-23]. Overuse of the saline solution may cause a hyperchloremic metabolic acidosis with related multiple adverse effects on metabolic and enzymatic function. These complications include decreased myofibril contractility and altered membrane ion channel function with consequent systemic vasodilatation and hypotension, arrhythmias, increase of pulmonary vascular resistance, as well as reduction of cardiac output and catecholamine resistance. Additionally, overly aggressive fluid resuscitation can also alter glucose metabolism and oxygen availability [24]. In turn, colloids such as dextran, hydroxyethyl starch (HES) and albumin, are related with a different type of adverse effects. Their use is associated with a negative impact on hemocoagulation caused by antiplatelet effects of these solutions and dilution coagulopathy [22, 25]. Various clinical studies comparing the effects of CBF maintenance strategies have failed to associate favorable neurological outcomes with these therapies and actually suggest a five-fold increase in the incidence of adult respiratory distress syndrome (ARDS). This study indicates that treating elevated ICP with DC to improve CBF results in fewer complica- tions than catecholamine use and/or aggressive volume resuscitation to elevate MAP, CPP and ultimately CBF [21].

\section{Mechanical Ventilation}

Mechanical ventilation can be life-saving as it provides airway protection and optimization of gas exchange but it also has associated complications that can worsen prognosis. The principal adverse events include Acute Lung Injury (ALI) / ARDS, pulmonary edema, pneumonia, and ventilator-induced lung injury (VLI).

Mechanical hyperventilation can become a necessary treatment of elevated ICP during the initial acute management of elevated ICP or as a life-saving maneuver to treat acute brain herniation. However it's prolonged use is associated with tissue hypoxia, decreased efficacy in controlling ICP due to readjustment of intracranial acid-base balance, and worse outcome. The Brain Trauma Foundation recommendations clearly focus on prevention of hypoxia, maintenance of CPP around $70 \mathrm{~mm} \mathrm{Hg}$ as well as an ICP under the threshold value of $20 \mathrm{~mm} \mathrm{Hg}$.

The use of PEEP in patients at risk of developing intracranial hypertension has been widely debated as it may increase ICP. The additional expiratory pressure support furnished by PEEP is reflected in the central venous compartment, interfering with the refilling of the vena cava causing an increase in cerebral venous pressure [26]. Alternatively, PEEP can increase functional residual capacity and prevent atelectases with alveolar recruitment which can optimize gas exchange and reestablish a more favorable ventilation/perfusion relationship leading to $\mathrm{PaCO} 2$ reduction and a decrease in ICP [27]. Additionally, PEEP can be beneficial by lowering $\mathrm{O}_{2}$ toxicity risk secondary to lower $\mathrm{FiO}_{2}$ allowance [28]. This study demonstrated that the management of intracranial hypertension along with DC helped to avoid potentially hazardous hyperventilation-associated complications as hyperventilation was utilized significantly less in the DC group compared to the standard care group. Also, PEEP was better tolerated in the DC group allowing for potential benefits such as decreased lung infection risk without concern for increased ICP during DC- induced ICP normalization.

\section{Neuroprotection}

One of the most frequently employed techniques for neuroprotection in patients with elevated ICP is barbiturate coma. Despite controversies regarding the ability of barbiturate coma to improve the long-term neurological outcome in patients with intractable intracranial hypertension, it still serves as a last resort in this patient population. Currently, high-dose barbiturate administration is recommended to control elevated ICP refractory to maximum standard medical and surgical treatment [29]. The application of barbiturate coma should be limited to hemodynamically stable patients who have a margin of neurological recovery. Barbiturates (sodium thiopental is used most often) cause a dose dependent reduction of neuronal activity, $\mathrm{CBF}$ as well as neuronal metabolic $\mathrm{O}_{2}$ and glucose requirements. As barbiturate administration increases, there is a corresponding increase in intracellular chlorine influx via gamma aminobutyric acid (GABA)-A receptors and blockade of AMPA receptors. The net effect translates to neuronal hyperpolarization and a major and diffuse depression of neuronal activity witnessed by electro-encephalographic variation [30]. The decrease in cerebral electrical activity is observed as burst suppression on EEG; thus constant monitoring 
is mandatory until the minimal effective barbiturate infusion dose is confirmed [31]. Potential adverse effects include: myocardial depression, peripheral vasodilatation with hypotension, a reduction in venous jugular oxygen saturation, immunosuppression, and leucopenia with increased incidence of infection [32]. Our results showed that DC decreased the necessity of barbiturate coma, again avoiding potential negative effects associated with adjunct therapies.

\section{Complications}

There are several complications associated with DC. Preoperative risk is primarily tied to the patient's clinical condition. Post-procedural complications can be early or late and should be considered by every intensivist concerned with neuro-resuscitation.

\section{Early Complications}

Bleeding can start during the surgical procedure and continue into the immediate postoperative period causing hematoma, cerebral edema and onset of relentless elevation of ICP. There is a $3-5 \%$ incidence of hematoma and bleeding [33]. External cerebral herniation can occur as well. This complication frequently develops within the first days that follow DC and is caused by insufficient resection to accommodate post-procedural brain expansion. Compression of the venous vasculature in the herniated cortex can lead to hemorrhagic infarction of the herniated tissue [33]. Additionally, increased compliance of cerebral tissue with room to expand following DC causes an increase in the hydrostatic gradient which can increase interstitial edema. An imbalance in CSF circulation also can lead to the formation of a subdural hygroma ipsilateral to the craniectomy [34].

\section{Late Complications}

There is a greater incidence of postoperative infection including meningitis and subdural empyema following DC compared to other neurosurgical procedures. The procedure can lead to lesions of the superficial temporal artery with a negative impact on scalp perfusion and wound scarring. Furthermore, the initial DC obligates a second surgical intervention in order to replace the removed bone, adding additional risks [35]. Formation of an obstructive hydrocephalus is another possible complication of DC, as well as the "Trephine" syndrome, consisting of a contralateral (to the DC) upper limb paresis developing months after the surgery. It may be caused by a contusion of the cerebral parenchyma and an anomalous CSF circulation that follows the DC. The symptoms generally revert with bone repositioning and adequate physiotherapy [36].

\section{Conclusions}

Clinical priorities in elevated ICP management are the maintenance of CPP along with adequate oxygen delivery to prevent secondary hypoxic damage. In such patients with elevated ICP, DC is an effective way to normalize ICP levels and re-establish optimal cerebral perfusion while reducing the need for aggressive medical therapies and associated cardiovascular and pulmonary complications. Furthermore, patients treated with DC are less likely to need neuro-protective techniques such as barbiturate coma. Neurological outcome in patients treated with DC was found to be better compared with patients receiving only medical treatment.
DC redefines clinical priorities for the management of patients at risk for intracranial hypertension; shifting from cerebral herniation avoidance to prevention of secondary cerebral damage. Such a treatment should be considered in all patients with elevated ICP refractive to initial conservative management and in those at risk for developing high intractable ICP.

\section{References}

[1]. Marmarou A, Anderson RL (1991) Impact of ICP instability and hypertension on outcome in patients with severe head trauma. J Neurosurg, 75: 159-66

[2]. Cremer OL, van Dijk GW, van Wensen E, Brekelmans GJ, Moons KG, et al. (2005) Effect of intracranial pressure monitoring and targeted intensive care on functional outcome after severe head injury. Crit Care Med, 33(10): 2207-13

[3]. Steiner LA, Andrews PJD (2006) Monitoring the injured brain: ICP and CBF. Br J Anaesth, 97(1): 26-38

[4]. Gwinnut CL, Saha B (2005) Cerebral flow and intracranial pressure. Anesth Intens Car Med, 6(5): 153-56

[5]. Dirnalg U, Pulsinelli W (1990) Autoregulation of cerebral blood flow in experimental focal brain ischemia. J Cereb Blo Flo Met, 10: 327-36

[6]. Bratton SL, Chestnut RM, Ghajar J (2007) Guidelines for the management of severe traumatic brain injury. VI. Indications for intracranial pressure monitoring. J Neurotrauma 24 Suppl., 1: S37-44

[7]. Thompson HJ, Kirkness CJ, Mitchell PH (2007) Intensive care unit management of fever following traumatic brain injury. Intens Crit Car Nurs, 23(2): $91-6$

[8]. Bilotta F, Caramia R, Paoloni FP, Delfini R, Rosa G (2009) Safety and efficacy of intensive insuline therapy in critical neurosurgical patients. Anesthesiology, 110(3): 611-19

[9]. Ghajar J, Hariri RJ, Narayan RK, Iacono LA, Firlik K, et al (1995) Survey of critical care management of comatose, head-injured patients in the United States. Crit Car Med, 23(3): 560-67.

[10]. Hutchinson P, Timofeev I, Kirkpatrick P (2007) Surgery for brain edema. Neurosurg Focus, 22(5): E14.

[11]. Tomofeev I, Hutchinson P (2006) Outcome after surgical decompression of severe traumatic brain injury. Injury, 37: 1125-32.

[12]. Sahuquillo J, Arikan F (2006) Decompressive craniectomy for the treatment of refractory high intracranial pressure in traumatic brain injury. Cochrane Database Syst Rev, 1: 93-100.

[13]. Williams RF, Magnotti LJ, Croce MA (2009) Impact of decompressive craniectomy on functional outcome after severe traumatic brain injury. J Trauma, 66:1570-76

[14]. Cooper PR, Rovit RL, Ransohoff J (1976) Hemicraniectomy in the treatment of acute subdural hematoma: a re-appraisal. Surg Neurol, 5: 25-8

[15]. Jennett B, Bond M (1975) Assessment of outcome after severe brain damage. Lancet, 1: 480-84

[16]. Daboussi A, Minville V, Leclerc-Foucras S, Geeraerts T, Esquerré JP, et al. (2009) Cerebral hemodynamic changes in severe head injury patients undergoing decompressive craniectomy. J Neurosurg Anesthesiol, 21: 339-345

[17]. Miller JD, Dearden NM, Piper IR, Chan KH (1992) Control of intracranial pressure in patients with severe head injury. J Neurotrauma, 9 (Suppl. 1): S317-S326,

[18]. Chambers IR, Treadwell L, Mendelow AD (2001) Determination of threshold levels of cerebral perfusion pressure and intracranial pressure in severe head injury by using receiver-operating characteristic curves: an observational study in 291 patients. J Neurosurg, 94: 412-416

[19]. White H, Venkatesh B (2008) Cerebral perfusion pressure in neuro trauma: a review. Neurosurg Anesthesiol, 107: 979-88

[20]. Clifton GL, Miller ER, Choi SC, Levin HS (2002) Fluids thresholds and outcome from severe brain injury. Crit Care Med, 30: 739-45

[21]. Contant CF, Valadka AB, Gopinath SP, Hannay HJ, Robertson CS (2001) Adult respiratory distress syndrome: a complication of induced hypertension after severe head injury. J Neurosurg, 95: 560-8

[22]. Hartog CS, Bauer M, Reinhart K (2011) The efficacy and safety of colloid resuscitation in the critically ill. Anesth Analg, 112(1): 156-64

[23]. Bindoli, A, Rigobello, M. P, Deeble, D. J (1992) Biochemical and toxicological properties of the oxidation products of catecholamines. Free Radical Biol. Med, 13: 391-405

[24]. Salim L(2007) Metabolic acidosis. Acta Med Indones-Indones J Intern Med, 39(3): $145-50$

[25]. Bilotta F, Rosa G (2007) Saline or Albumin for Fluid resuscitation in Traumatic Brain Injury (Letter to the Editor). N Eng J Med, 357 (25): 2634

[26]. Pick RA, Handler JB, Friedman AS (1982) The cardiovascular effects of PEEP. Chest, 3: 345-350 
[27]. Koehler RC, Michael JR (1985)Transmission of intrathoracic pressure to intracranial pressure. Clin Crit Care, 1: 212-214

[28]. Bratton SL, Chestnut RM, Ghajar J (2007) Guidelines for the management of severe traumatic brain injury. VI. Hyperventilation. J Neurotrauma, 24 Suppl 1: S87-90

[29]. Bratton SL, Chestnut RM, Ghajar J (2007) Guidelines for the management of severe traumatic brain injury. VI. Anesthetics, analgesics and sedatives. J Neurotrauma, 24 Suppl 1: S71-76

[30]. Stover JF, Lenzlinger PM, Stocker R, Morganti-Kossmann MC, Imhof HG, et al (1998) Thiopental in CSF and serum correlates with prolonged loss of cortical activity. Eur Neurol, 39: 223-28

[31]. Claasen J, Mayer S (2002) Continuous electroencephalographic monitoring in neurocritical care. Curr Neurol Neurosci Rep, 2: 534-540

[32]. Stover JF, Stocker R (1998) Barbiturate coma may promote reversible bone marrow suppression in patients with severe isolated traumatic brain injury. Eur J Clin Pharmacol, 54: 529 -34

[33]. Flint AC, Manley GT, Gean AD, Hemphill JC III, Rosenthal G (2008) Post-operative expansion of hemorrhagic contusions after unilateral decompressive hemicraniectomy in severe traumatic brain injury. J Neurotrauma, 25: $503-512$

[34]. Lang JK, Ludwig HC, Mursch K, Zimmerer B, Markakis E (1999) Elevated cerebral perfusion pressure and low colloid osmotic pressure as a risk factor for subdural space-occupying hygromas? Surg Neurol, 52: 630-637

[35]. Marmarou A, Anderson RL (1991) Impact of ICP instability and hypertension on outcome in patients with severe head trauma. J Neurosurg, 75: 159-66

[36]. Stiver SI (2009) Complications of decompressive craniectomy for traumatic brain injury. Neurosurg Focus, 26(6): E7 